The Free Internet Journal for Organic Chemistry
Paper

Arkivoc 2018, part iv, 183-194

\title{
A formal approach to the cyanobacterial sunscreen indole, prenostodione
}

\author{
Ilene L. Green, Jason J. Jordan, and Jeanese C. Badenock* \\ Department of Biological and Chemical Sciences, University of the West Indies, Cave Hill, Barbados \\ Email: jeanese.badenock@cavehill.uwi.edu
}

Dedicated to Dr. Gordon W. Gribble in recognition of his outstanding contributions to the field of indole chemistry

Received 12-31-2017

Accepted 03-15-2018

Published on line 04-07-2018

\section{Abstract}

The synthesis of the indole sunscreen pigment prenostodione was attempted via an LDA-initiated condensation of $\mathrm{N}$-carbamate indole-2-methyl ester 22 with 4-[(t-butyldimethylsilyl)oxy]benzaldehyde (14) and a late-stage Vilsmeier-Haack formylation. Difficulties with the ensuing oxidation required installation of a C-3 carboxylic acid necessitating the use of a recently reported protocol and thus a formal synthesis of the natural product was realized from 2-aminobenzyl alcohol (17) in nine steps.<smiles>Nc1ccccc1CO</smiles>

17<smiles>CC(=O)C(=Cc1ccc(O)cc1)c1[nH]c2ccccc2c1C(=O)O</smiles>

3

Keywords: Prenostodione, isoprenostodione, scytonemin, cyanobacteria, Pinnick-Lindgren oxidation 


\section{Introduction}

The ubiquitous indole skeleton continues to feature prominently in alkaloids isolated from diverse terrestrial and marine sources. ${ }^{1-2}$ One such source of novel, and in many cases bioactive, indole-based isolates is cyanobacteria. ${ }^{3-5}$ These oxygenic photoautotrophic prokaryotes, commonly referred to as blue-green algae, date back as far as 3.5 billion years ${ }^{6}$ and adopt filamentous, unicellular, or aggregated morphologies generally reflective of the habitats to which they have adapted..$^{7-8}$

From those alkaloids reported in the last few decades, members of the classes of hapalindoles, ${ }^{9-21}$ fischerindoles, ${ }^{9,17-18,22}$ ambiguines, ${ }^{23,36-37}$ and welwitindolinones ${ }^{9,18,22,25-29}$ have received significant attention from the synthetic community, with over 10 groups rendering total or formal protocols - many with enantioselective precision - since their first isolation in $1984 .{ }^{30}$ The sunscreen indole pigments scytonemin (1), ${ }^{31}$ nostodione A (2), ${ }^{32-34}$ and prenostodione (3), ${ }^{33}$ isolated from a variety of cyanobacterial species including Scytonema sp., Nostoc sp., and Scytonema hofmanni, however, have not garnered the same level of interest. While a few total syntheses of these three species are available, ${ }^{35-39}$ the focus has been on the exploration of their bioactivity and their enzymatically determined biosynthetic pathways. ${ }^{40-48}$ More interestingly, the syntheses of four recently isolated and structurally-related derivatives - scytonine (4), dimethoxyscytonemin (5), tetramethoxyscytonemin (6), ${ }^{49}$ and scytonemin-3a-imine $(7)^{50}$ - remain unreported (Figure 1).

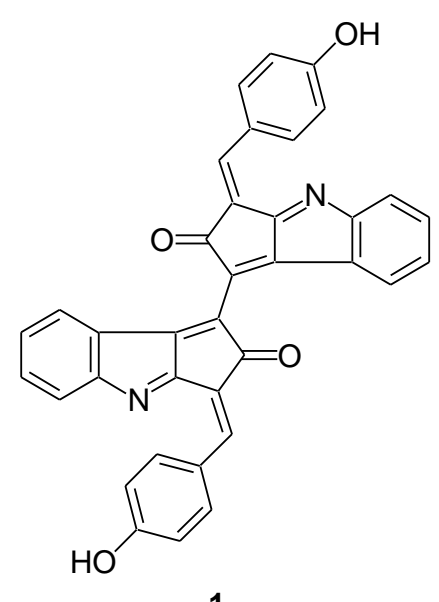

1

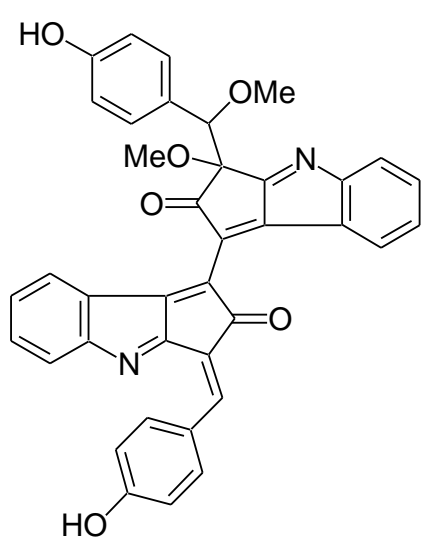

5<smiles>O=C1C(=O)C2=C(NC1=Cc1ccc(O)cc1)c1ccccc12</smiles><smiles>COC(=O)/C(=C/c1ccc(O)cc1)c1[nH]c2ccccc2c1C(=O)O</smiles>

2<smiles>COC(c1ccc(O)cc1)C1(OC)C(=O)C(C2=C3C(=Nc4ccccc43)C3(OC)C(=O)C2=C(c2ccc(O)cc2)C3=O)=C2N=C3C=CC=CC321</smiles>

6<smiles>COC(=O)C1=C(c2c(C(C)=O)[nH]c3ccccc23)c2c([nH]c3ccccc23)C1=O</smiles>

3

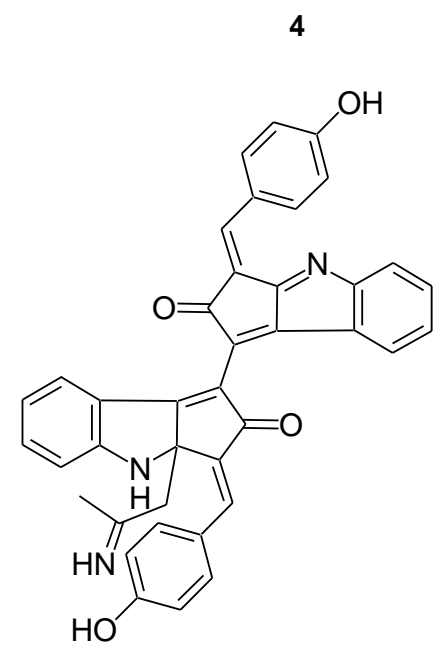

7

Figure 1. Cyanobacterial alkaloids of interest. 
Driven by our desire to access scytonemin (1) we sought an initial approach to prenostodione (3), proposed by Pluotno and Carmeli ${ }^{33}$ to be the precursor of both nostodione A (2) and scytonemin (1). This indole-3-carboxylic acid derivative is substituted with a $p$-hydroxybenzylidene group appended at the C-2 position of the indole and was determined to have E-geometry around the exocyclic double bond. We herein outline our endeavors towards this target compound along with the interesting detours which resulted in a synthesis of an isomer of the natural product, dubbed isoprenostodione (13). ${ }^{51}$

\section{Results and Discussion}

A previous account described our initial strategy, which was centered on the installation of the vinyl appendage at the methylene position of an indole diester, and revealed a correlation between the choice of ester and the geometry observed in the coupling. ${ }^{52}$ The indole methyl diester 10, accessed via a Fischer indole synthesis, was therefore smoothly converted into vinyl indole 12 after reaction with 4-[(TBS)oxy]benzaldehyde (14) in the presence of base - LDA then $\mathrm{CaH}_{2}$. A final selective hydrolysis, though not without precedent, ${ }^{53}$ proved problematic in this system and regrettably dimethyl ester $\mathbf{1 2}$ underwent cleavage at the C-2 position rather than at the intended C-3 site (Scheme 1).
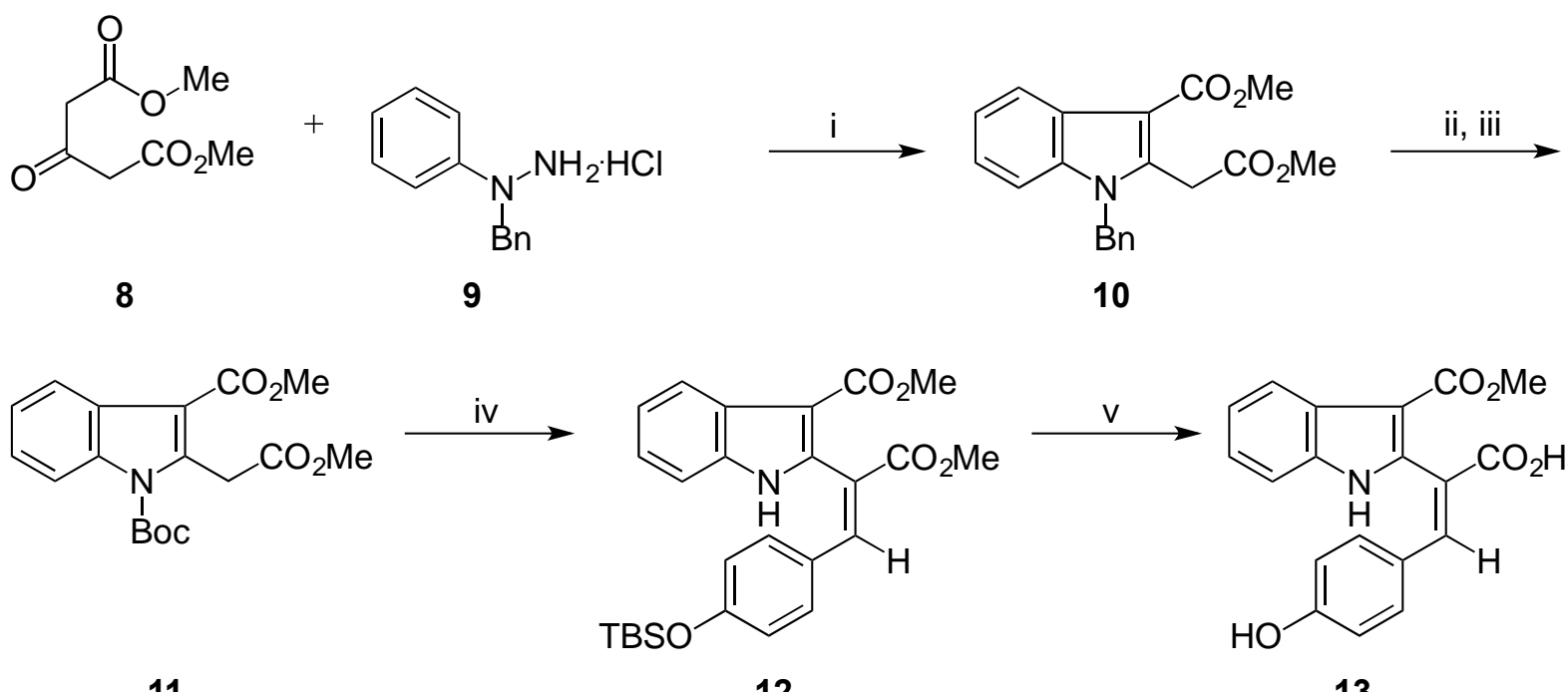

11

12

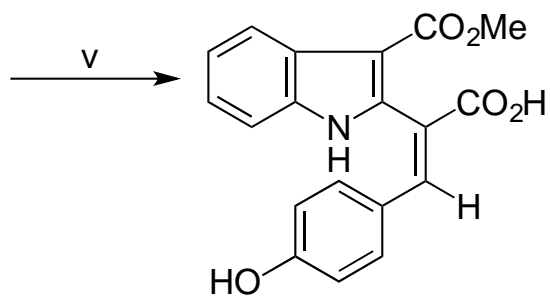

13

Scheme 1. Reagents and Conditions: i. $\mathrm{MeOH}, \Delta, 12 \mathrm{~h}(57 \%)$; ii. $\mathrm{AlCl}_{3}, \mathrm{PhH}, \Delta, 0.5 \mathrm{~h}(85 \%)$; iii. $\mathrm{Boc}_{2} \mathrm{O}, \mathrm{DMAP}$, THF, rt (100\%); iv. $n$-BuLi, $\left(\left(\mathrm{CH}_{3}\right)_{2} \mathrm{CH}\right)_{2} \mathrm{NH}, 4-\mathrm{TBSOC}_{6} \mathrm{H}_{4} \mathrm{CHO}(14),-78{ }^{\circ} \mathrm{C} ; \mathrm{CaH}_{2}, \Delta, 1 \mathrm{~h}(46 \%) ; \mathrm{v} . \mathrm{KOH}, \mathrm{MeOH}, \Delta$, $4 \mathrm{~h}(23 \%)$.

This disappointing result caused us to consider a new approach which was aimed at introducing the C-3 acid in the terminal stages of the synthesis. Furthermore, it was envisioned that, when used in tandem with the base-catalyzed coupling protocol ${ }^{54}$ used in our previous attempt at prenostodione (3), ${ }^{51}$ a late-stage Vilsmier-Haack formylation ${ }^{55}$ could introduce an oxidizable C-3 formyl group (Scheme 2). 


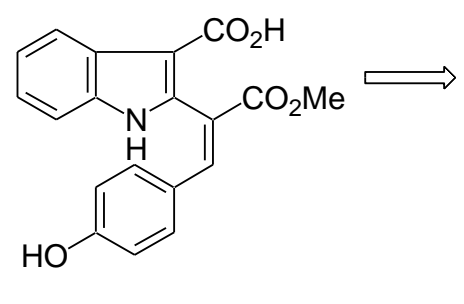

3

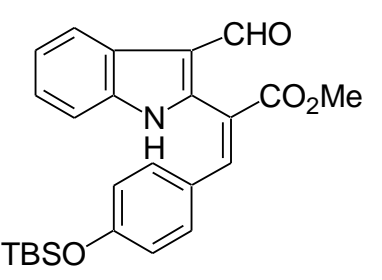

15

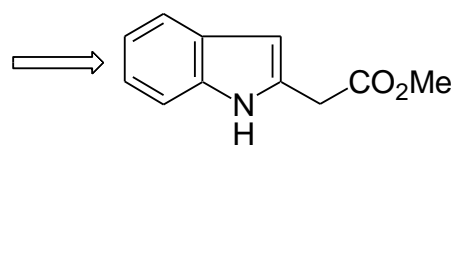

16

Scheme 2. Revised retrosynthetic strategy to prenostodione (3).

Consequently, treatment of 2-aminobenzyltriphenylphosphonium bromide (18), obtained from reaction of commercially obtained 2-aminobenzyl alcohol (17) and triphenylphosphonium hydrogen bromide, with methyl malonyl chloride, resulted in the isolation of 1,3-dicarbonyl intermediate 19 as a white powder in $79 \%$ yield. ${ }^{56}$ Construction of the indole ring was realized when 19 was treated with slightly more than stoichiometric amounts of potassium tert-butoxide resulting in indole ester $\mathbf{1 6}$ - the product of an intramolecular Wittig condensation (Scheme 3). ${ }^{57}$

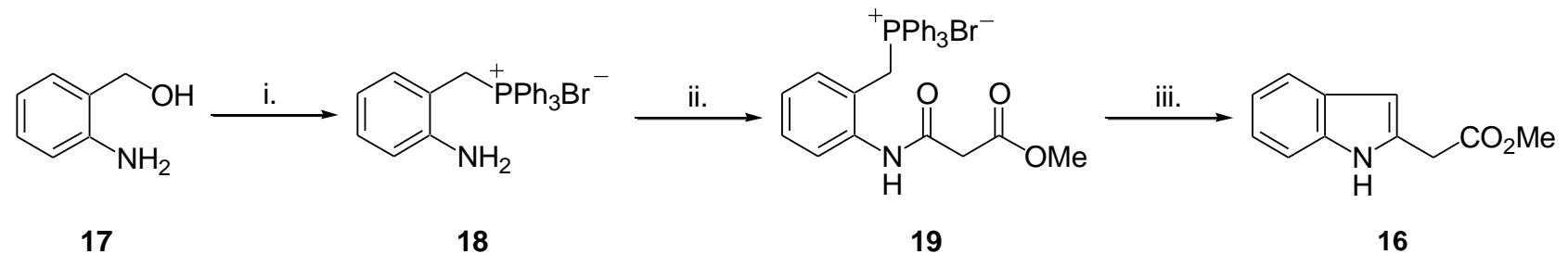

Scheme 3. Reagents and Conditions: i. $\mathrm{PPh}_{3} \bullet \mathrm{HBr}, \mathrm{CH}_{3} \mathrm{CN}(88 \%) ;$ ii. $\mathrm{MeO}_{2} \mathrm{CCH}_{2} \mathrm{COCl}, \mathrm{CH}_{2} \mathrm{Cl}_{2}, 3 \mathrm{~h}$ (79\%); iii. 1.1 eq. KOt-Bu, PhMe, $\Delta, 6 \mathrm{~h}$ (74\%).

With sights firmly set on accessing vinyl indole 15, a Vilsmeier Haack formylation of indole 16 resulted in 3formylindole derivative 20 but suffered from low yields after purification. Resultantly, and notwithstanding the successful conversion of $\mathbf{2 0}$ to the $\mathrm{N}$-Boc-indole $\mathbf{2 1}$, the order of the installation of the functional groups was re-engineered with a view to improving the product yields. Accordingly, coupling of $t$-butoxycarbamate 22, obtained by protection of indole 16 in almost quantitative yield, with 4-[(TBS)oxy]benzaldehyde (14), in the presence of LDA and $\mathrm{NaH}$, led to the isolation of alkene $\mathbf{2 3}$ as a yellow solid in $44 \%$ yield as outlined in Scheme 4. Although alkene $\mathbf{2 3}$ was observed to undergo rapid decomposition in deuterated chloroform, we were able to confirm its identity using NMR spectroscopy. Moreover, 2D NOESY and 1D NOE experiments, conducted on 23, did not reveal any correlation between the $\mathrm{NH}$ proton $(\delta 8.41)$ and the vinyl proton at $\delta 7.85$ and consequently gave support to the assignment of an E-geometry at the exocyclic double bond. ${ }^{58}$

The introduction of the C-3 aldehyde via Vilsmeier-Haack formylation, this time in the absence of an alkali in the hydrolysis step, ${ }^{59}$ also resulted in the cleavage of the silyl ether functional group and therefore completion of the strategy would have only required oxidation of the aldehyde to a carboxylic acid (Scheme 5). Unfortunately, a carboxylic acid did not result from reaction under Pinnick-Lindgren oxidation conditions, ${ }^{60,61}$ nor upon using other oxidants such as $\mathrm{AgNO}_{3}, \mathrm{KMnO}_{4}$, and $\mathrm{DDQ}$. During the ensuing lull in progression, we were fatefully made aware of a recent paper by Biswas et al. which outlined the conversion of methyl 2-(3-formyl-1H-indole-2-yl)acetate into prenostodione (3). ${ }^{37}$ Their approach, while synthetically unmatched, was strikingly similar to our proposed route and involved the coupling of methyl ester aldehyde $\mathbf{2 0}$ with $p$-hydroxybenzaldehyde, in the presence of the catalyst L-proline, to generate aldehyde $\mathbf{2 4}$. There was 
also a notable absence of a direct oxidation protocol which was indicative of the incompatibility of the indole $\mathrm{NH}$ and/or the phenol $\mathrm{OH}$ with oxidation conditions.

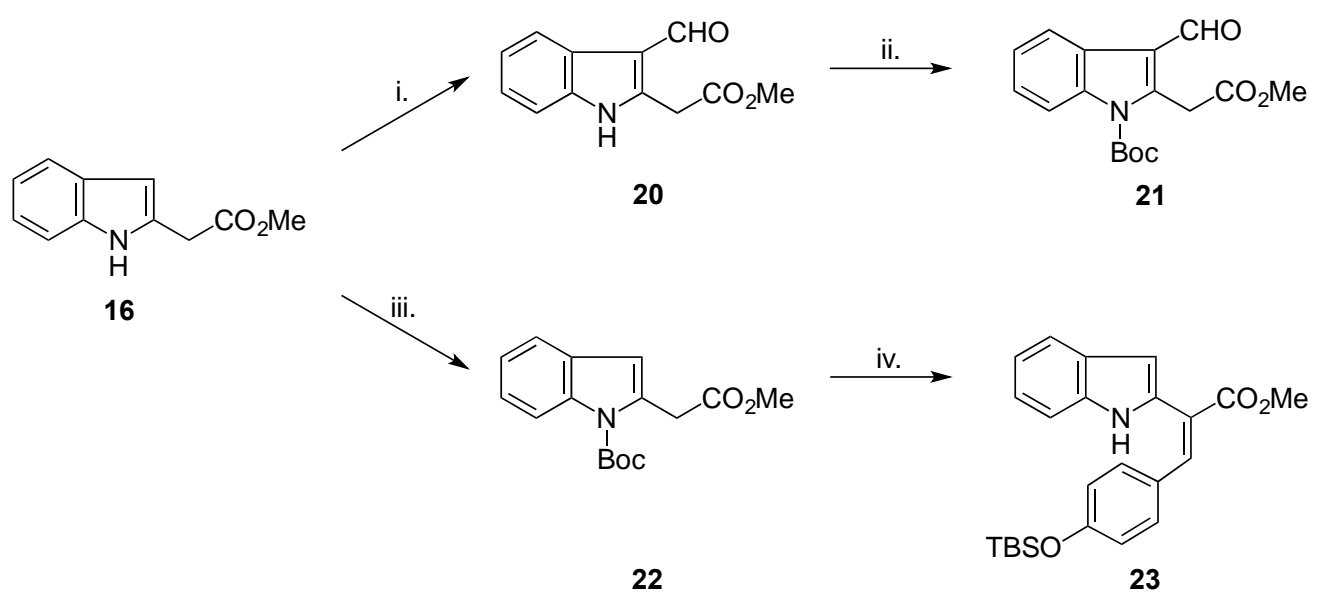

Scheme 4. Reagents and Conditions: i. $\mathrm{POCl}_{3}, \mathrm{DMF}, 0{ }^{\circ} \mathrm{C}, 1 \mathrm{~h} ; \mathrm{NaHCO}_{3}, \mathrm{NaOH}(33 \%)$; ii. $\mathrm{Boc}_{2} \mathrm{O}, \mathrm{DMAP}, \mathrm{THF}, \mathrm{rt}$ (65\%); iii. $\mathrm{Boc}_{2} \mathrm{O}, \mathrm{DMAP}$, THF, rt (96\%); iv. LDA, 4-TBSOC ${ }_{6} \mathrm{H}_{4} \mathrm{CHO}(14)$, THF $-78{ }^{\circ} \mathrm{C}, 1 \mathrm{~h} ; \mathrm{NaH}, \Delta, 2 \mathrm{~h}(44 \%)$.

Given this regretful turn of events, we decided to complete a formal synthesis of prenostodione (3) using this reported protocol ${ }^{37}$ and reprotected both the $-\mathrm{NH}$ and $-\mathrm{OH}$ functional groups, by stirring aldehyde 24 with 2.5 equivalents of $\mathrm{Boc}_{2} \mathrm{O}$ in the presence of catalytic amounts of DMAP at room temperature for 4 hours (Scheme 5). The ensuing Pinnick-Lindgren oxidation required the sequential addition of sodium chlorite and monosodium phosphate to the heterogeneous mixture of aldehyde 25 and sulfamic acid in $t-\mathrm{BuOH} / \mathrm{H}_{2} \mathrm{O}$, and afforded di-Boc-acid $\mathbf{2 6}$ as a pale yellow solid in $\mathbf{8 7 \%}$ yield (over 2 steps). The identity of $\mathbf{2 6}$ was confirmed by the disappearance of the aldehyde signals found at $\delta 9.81$ and $\delta 186.7$ in ${ }^{1} \mathrm{H}$ - and ${ }^{13} \mathrm{C}$-NMR spectra, respectively. In a final effort to secure the coveted natural product prenostodione (3), diBoc-acid 26 was treated with TFA in $\mathrm{CH}_{2} \mathrm{Cl}_{2}$ at $0{ }^{\circ} \mathrm{C}$ to facilitate deprotection and the natural product was obtained as a yellow oil, albeit in a modest $24 \%$ yield. The NMR spectra of the synthetic product were comparable to literature values for the natural product but revealed minor deviations from those of isoprenostodione (13) previously reported (Table 1). ${ }^{33}$

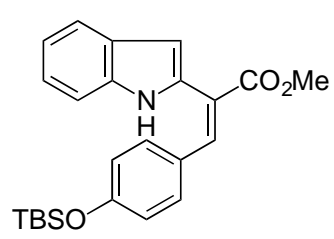

23

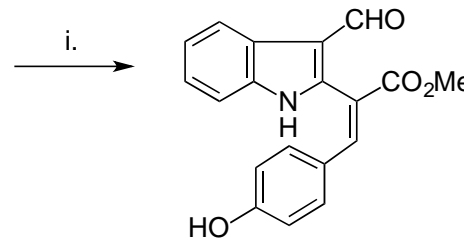

24

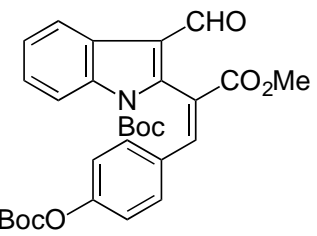

25
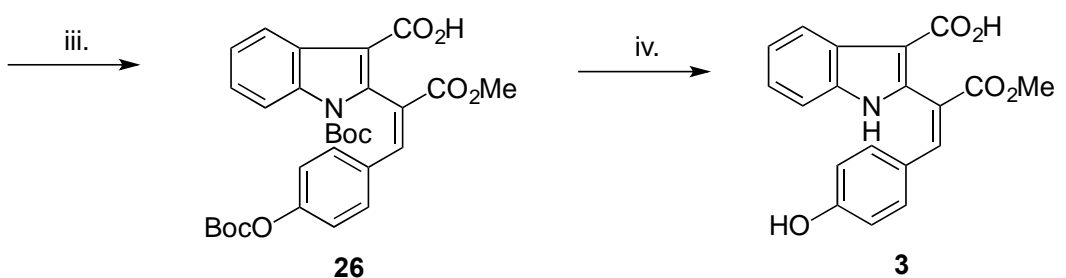

Scheme 5. Reagents and Conditions: i. $\mathrm{POCl}_{3}, \mathrm{DMF}, \Delta, 1 \mathrm{~h}(79 \%)$; ii. $\mathrm{Boc}_{2} \mathrm{O}, \mathrm{DMAP}, \mathrm{CH}_{2} \mathrm{Cl}_{2}, \mathrm{rt}(100 \%)$; iii. $\mathrm{NH}_{2} \mathrm{SO}_{3} \mathrm{H}, \mathrm{NaClO}_{2}, \mathrm{NaH}_{2} \mathrm{PO}_{4}, t-\mathrm{BuOH} / \mathrm{H}_{2} \mathrm{O}(3: 1)$, rt, $8 \mathrm{~h}(87 \%)$; iv. TFA, $\mathrm{CH}_{2} \mathrm{Cl}_{2}, 0^{\circ} \mathrm{C}, 8 \mathrm{~h}(24 \%)$. 
Table 1. Comparison of ${ }^{1} \mathrm{H}$ - and ${ }^{13} \mathrm{C}-\mathrm{NMR}$ data for the naturally occurring prenostodione (3) (N.P.), synthetic prenostodione (S.P.) and isoprenostodione (13) (I.P) $)^{33,51}$
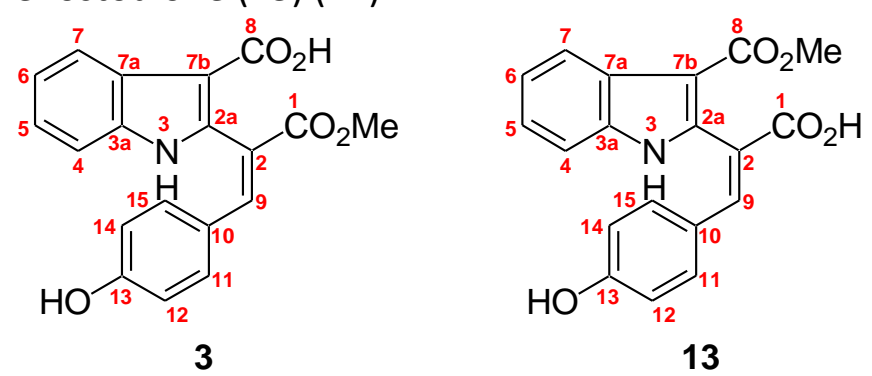

\begin{tabular}{|c|c|c|c|c|c|c|}
\hline Position & N.P. $\left(\delta_{C}\right)^{a}$ & S.P. $\left(\delta_{C}\right)^{b}$ & I.P. $\left(\delta_{C}\right)^{a}$ & N.P. $\left(\delta_{H}\right)^{a}$ & S.P. $\left(\delta_{H}\right)^{b}$ & I.P. $\left(\delta_{H}\right)^{a}$ \\
\hline 1 & 166.9 & 166.8 & 167.8 & & & \\
\hline 1-OMe & 52.1 & 52.0 & 50.8 & $3.63 \mathrm{~s}$ & $3.64 \mathrm{~s}$ & $3.69 \mathrm{~s}$ \\
\hline 2 & 120.4 & 120.3 & 120.5 & & & \\
\hline $2 a$ & 139.3 & 139.2 & 140.9 & & & \\
\hline 3 & & & & $11.82 \mathrm{~s}$ & 11.84 & $11.93 \mathrm{~s}$ \\
\hline $3 a$ & 135.8 & 135.7 & 135.8 & & & \\
\hline 4 & 112.1 & 112.0 & 112.2 & $\begin{array}{c}7.37 \mathrm{~d} \\
(8.8 \mathrm{~Hz})\end{array}$ & $\begin{array}{c}7.37-7.38 \mathrm{~d} \\
(6.7 \mathrm{~Hz})\end{array}$ & $\begin{array}{c}7.38-7.41 \\
m\end{array}$ \\
\hline 5 & 122.5 & 122.4 & 122.8 & $7.20 \mathrm{~m}$ & $7.18-7.20$ & $7.22-7.18 d$ \\
\hline 6 & 121.2 & 121.1 & 121.1 & $7.18 \mathrm{~m}$ & $\mathrm{~m}$ & $(8.0 \mathrm{~Hz})$ \\
\hline 7 & 121.2 & 121.1 & 121.6 & $\begin{array}{c}8.03 \mathrm{~d} \\
(8.7 \mathrm{~Hz})\end{array}$ & $\begin{array}{c}8.04-8.05 \mathrm{~d} \\
(6.7 \mathrm{~Hz})\end{array}$ & $\begin{array}{c}8.03-8.00 \mathrm{~d} \\
(8.7 \mathrm{~Hz})\end{array}$ \\
\hline $7 a$ & 127.1 & 127.0 & 126.7 & & & \\
\hline $7 b$ & 105.8 & 105.7 & 104.8 & & & \\
\hline 8 & 165.8 & 165.7 & 165.0 & & & \\
\hline 9 & 142.3 & 142.1 & 141.9 & $7.78 \mathrm{~s}$ & $7.79 \mathrm{~s}$ & $7.77 \mathrm{~s}$ \\
\hline 10 & 124.8 & 124.7 & 125.1 & & & \\
\hline 11,15 & 132.3 & 132.2 & 132.2 & $\begin{array}{c}6.84 \mathrm{~d} \\
(8.7 \mathrm{~Hz})\end{array}$ & $\begin{array}{l}6.84-6.85 \\
d(8.3 \mathrm{~Hz})\end{array}$ & $\begin{array}{c}6.84-6.81 \mathrm{~d} \\
(8.6 \mathrm{~Hz})\end{array}$ \\
\hline 12,14 & 115.8 & 115.6 & 115.8 & $\begin{array}{c}6.58 \mathrm{~d} \\
(8.7 \mathrm{~Hz})\end{array}$ & $\begin{array}{c}6.58-6.59 \mathrm{~d} \\
(8.3 \mathrm{~Hz})\end{array}$ & $\begin{array}{c}6.60-6.57 \mathrm{~d} \\
(8.6 \mathrm{~Hz})\end{array}$ \\
\hline 13 & 159.6 & 159.4 & 159.4 & & & \\
\hline $13-0$ & & & & $9.99 \mathrm{~s}$ & $10.05 \mathrm{~s}$ & $10.04 \mathrm{~s}$ \\
\hline
\end{tabular}

${ }^{a} \mathrm{NMR}$ experiments conducted in DMSO- $\mathrm{d}_{6} ;{ }^{\mathrm{b}} \mathrm{NMR}$ experiments conducted in $\mathrm{CDCl}_{3}$ 


\section{Conclusions}

We have completed a formal synthesis of prenostodione (3) in nine steps from commercially available 2aminobenzyl alcohol. Efforts geared towards improving the yield of the hydrolysis step and developing synthesis of other similar indole pigments continue in our laboratory.

\section{Experimental Section}

General. Melting points were determined on a Sanyo Gallenkamp capillary melting point apparatus, in open capillaries and are uncorrected. Thin layer chromatography (TLC) was performed on Whatman brand $20 \times 20$ $\mathrm{cm}$ aluminum backed silica plates with fluorescent indicator. Plates were visualized by $254 \mathrm{~nm}$ UV light. Flash chromatography was carried out using Silicycle ultra-pure silica gel $60 \AA$ (230 - 400 mesh). Preparative TLC (PTLC) was performed with Merck precoated TLC plates silica gel $60 \mathrm{~F}_{254} \cdot{ }^{1} \mathrm{H}(300 \mathrm{MHz}),{ }^{1} \mathrm{H}(600 \mathrm{MHz}),{ }^{13} \mathrm{C}(75$ $\mathrm{MHz}),{ }^{13} \mathrm{C}(150 \mathrm{MHz}) \mathrm{NMR}$ spectra were recorded on Bruker-300 and -600 Fourier transform spectrometers. The chemical shifts are reported in $\delta(\mathrm{ppm})$ using the $\delta 7.26$ signal of $\mathrm{CDCl}_{3}\left({ }^{1} \mathrm{H}-\mathrm{NMR}\right)$ and the $\delta 77.16$ signal of $\mathrm{CDCl}_{3}\left({ }^{13} \mathrm{C}-\mathrm{NMR}\right)$, the $\delta 2.50$ signal of $\left(\mathrm{CD}_{3}\right)_{2} \mathrm{SO}\left({ }^{1} \mathrm{H}-\mathrm{NMR}\right)$ and the $\delta 39.50$ signal of $\left(\mathrm{CD}_{3}\right)_{2} \mathrm{SO}\left({ }^{13} \mathrm{C}-\mathrm{NMR}\right)$. Ultraviolet (UV) spectra were recorded on a Hewlett-Packard 8451A Diode Array UV spectrophotometer and are reported in nanometers. Infrared spectra (IR) were recorded on a Shimadzu IR Affinity-1 FTIR spectrophotometer and are referenced to the $1601 \mathrm{~cm}^{-1}$ band of polystyrene. IR spectra were obtained using solid potassium bromide pellets $(\mathrm{KBr})$ and are reported in reciprocal centimeters.

[2-(Methoxycarbonylacetamido)benzyl]triphenylphosphonium bromide (19). Methyl malonyl chloride (1.02 $\mathrm{mL}, 1.29 \mathrm{~g}, 9.48 \mathrm{mmol}, 1 \mathrm{eq}$.) was added to a stirring solution of 2-aminobenzyltriphenylphosphonium bromide (18) $\left(4.12 \mathrm{~g}, 9.48 \mathrm{mmol}, 1\right.$ eq.) in $\mathrm{CH}_{2} \mathrm{Cl}_{2}(20 \mathrm{~mL})$. After $3 \mathrm{~h}$, the solvent was removed and the residue was recrystallized from hot $\mathrm{MeOH}$ to give phosphonium bromide $19(4.10 \mathrm{~g}, 79 \%)$ as a white solid; $\mathrm{mp} 235^{\circ} \mathrm{C}$ (dec) (lit. $\left.{ }^{62} \mathrm{mp} 238-239{ }^{\circ} \mathrm{C}\right) ;{ }^{1} \mathrm{H}-\mathrm{NMR}\left(300 \mathrm{MHz}, \mathrm{CDCl}_{3}\right) \delta 10.44(\mathrm{~s}, 1 \mathrm{H}), 7.60-7.80(\mathrm{~m}, 17 \mathrm{H}), 6.81-6.86(\mathrm{~m}, 1 \mathrm{H})$, 6.70-6.74 (m, 1H), 5.46-5.51 (d, J 14.4 Hz, 2H), $3.65(\mathrm{~s}, 3 \mathrm{H}), 3.52(\mathrm{~s}, 2 \mathrm{H}) ;{ }^{13} \mathrm{C}-\mathrm{NMR}\left(75 \mathrm{MHz}, \mathrm{CDCl}_{3}\right) \delta 168.6$, $165.5,138.0,135.2,134.4,131.6,130.3,129.3,127.2,125.3,120.1,118.4,117.3,52.1,43.4 . \mathrm{IR} v$ (KBr) 3433, $3101,1744,1682,1435,1242,1157,1111,748 \mathrm{~cm}^{-1}$; UV $\lambda_{\max }(\mathrm{MeOH}) 204,241,292 \mathrm{~nm}$.

Methyl 2-(1H-indol-2-yl)acetate (16). Potassium tert-butoxide $(835 \mathrm{mg}, 7.44 \mathrm{mmol}, 1.1 \mathrm{eq}$.) was added to a stirring suspension of phosphonium bromide $19(3.71 \mathrm{~g}, 6.77 \mathrm{mmol}, 1$ eq.) in toluene $(17 \mathrm{~mL})$ at reflux. After 6 $h$, the reaction was poured onto $\mathrm{H}_{2} \mathrm{O}$ and stirred for a further $10 \mathrm{~min}$. The mixture was extracted with $\mathrm{CH}_{2} \mathrm{Cl}_{2}$ $(3 \times 30 \mathrm{~mL})$ and the organic extracts were combined, washed with brine $(1 \times 30 \mathrm{~mL})$, dried $\left(\mathrm{MgSO}_{4}\right)$ and concentrated in vacuo. Purification by flash chromatography (2:1 hexanes : EtOAc) gave the desired indole 16 (947 mg, 74\%) as a pale yellow solid: $\mathrm{mp} 65-67^{\circ} \mathrm{C}$ (lit. $\left.{ }^{62} \mathrm{mp} 68-69^{\circ} \mathrm{C}\right) ;{ }^{1} \mathrm{H}-\mathrm{NMR}\left(300 \mathrm{MHz}, \mathrm{CDCl}_{3}\right) \delta 8.64(\mathrm{~s}, 1 \mathrm{H})$, 7.54-7.56 (d, $1 \mathrm{H}, J 7.74 \mathrm{~Hz}), 7.34-7.37(\mathrm{~d}, 1 \mathrm{H}, J 7.98 \mathrm{~Hz}), 7.06-7.19(\mathrm{~m}, 2 \mathrm{H}), 6.36(\mathrm{~s}, 1 \mathrm{H}), 3.85(\mathrm{~s}, 2 \mathrm{H}), 3.76(\mathrm{~s}$, $3 \mathrm{H}) ;{ }^{13} \mathrm{C}-\mathrm{NMR}\left(75 \mathrm{MHz}, \mathrm{CDCl}_{3}\right) \delta 171.1,136.5,130.5,128.3,121.9,120.3,120.0,110.9,102.0,52.5,33.9$; IR v (KBr) 3356, 2847, 1728, 1543, $748 \mathrm{~cm}^{-1}$; UV $\lambda_{\max }(\mathrm{MeOH}) 219,271,290,389 \mathrm{~nm}$.

tert-Butyl 2-(2-methoxy-2-oxoethyl)-1H-indole-1-carboxylate (22). To a solution of indole 16 (499 mg, 2.63 mmol, 1 eq.) in dry THF (17 mL) was added DMAP (14.9 mg, $0.122 \mathrm{mmol}, 0.05$ eq.) and di-tert-butyl dicarbonate $(637 \mathrm{mg}, 2.92 \mathrm{mmol}, 1.1 \mathrm{eq}$.) with stirring. The reaction was allowed to stir at rt overnight before being poured onto ice $\mathrm{H}_{2} \mathrm{O}(20 \mathrm{~mL})$. The mixture was extracted with $\mathrm{CH}_{2} \mathrm{Cl}_{2}(3 \times 20 \mathrm{~mL})$ and the organics were combined, dried $\left(\mathrm{MgSO}_{4}\right)$ and concentrated in vacuo. Purification by column chromatography (1:2 hexanes : 
EtOAc) gave the protected indole $22(0.735 \mathrm{~g}, 96 \%)$ as a pale yellow solid: $\mathrm{mp} 52-55{ }^{\circ} \mathrm{C} ;{ }^{1} \mathrm{H}-\mathrm{NMR}(300 \mathrm{MHz}$, $\left.\mathrm{CDCl}_{3}\right) \delta 8.07-8.10(\mathrm{~d}, \mathrm{~J} 7.8 \mathrm{~Hz}, 1 \mathrm{H}), 7.46-7.49(\mathrm{~m}, 1 \mathrm{H}), 7.16-7.29(\mathrm{~m}, 2 \mathrm{H}), 6.45(\mathrm{~s}, 1 \mathrm{H}), 4.03(\mathrm{~s}, 2 \mathrm{H}), 3.69(\mathrm{~s}, 3 \mathrm{H})$, $1.64(\mathrm{~s}, 9 \mathrm{H}) ;{ }^{13} \mathrm{C}-\mathrm{NMR}\left(75 \mathrm{MHz}, \mathrm{CDCl}_{3}\right) \delta 170.9,150.6,136.7,133.3,128.9,124.1,122.9,120.4,115.8,110.5$, 84.4, 52.1, 36.3, 28.2; IR $v(\mathrm{KBr}) 3449,1736,1381,1327,748 \mathrm{~cm}^{-1}$; UV $\lambda_{\max }(\mathrm{MeOH}) 259,282 \mathrm{~nm}$.

(E)-Methyl 3-(4-((tert-butyldimethylsilyl)oxy)phenyl)-2-(1H-indol-2-yl)acrylate (23). To a stirred solution of LDA (0.74 mL, $1.50 \mathrm{mmol}, 1.5$ eq., $2.0 \mathrm{M}$ in hexanes) in dry THF (4 mL) at $-75{ }^{\circ} \mathrm{C}$ was added a solution of ester $22(286 \mathrm{mg}, 0.99 \mathrm{mmol}, 1$ eq.) in dry THF $(4 \mathrm{~mL})$. The mixture stirred for $45 \mathrm{~min}$ before adding a solution of siloxy benzaldehyde 14 (260 mg, $1.10 \mathrm{mmol}, 1.1$ eq.) in dry THF (2 mL) and further stirred for $1 \mathrm{~h}$ at $-75{ }^{\circ} \mathrm{C}$ before allowing the mixture to warm to rt slowly. $\mathrm{NaH}(27.8 \mathrm{mg}, 1.2 \mathrm{mmol}, 1.2 \mathrm{eq}$.) was added and the reaction mixture was stirred at reflux for $1 \mathrm{~h}$. The solution was allowed to cool and poured onto $\mathrm{H}_{2} \mathrm{O}(25 \mathrm{~mL})$ with stirring. The aqueous mixture was extracted with $\mathrm{CH}_{2} \mathrm{Cl}_{2}(3 \times 25 \mathrm{~mL})$, and the combined organic extracts were washed with brine $(1 \times 25 \mathrm{~mL})$, dried $\left(\mathrm{MgSO}_{4}\right)$ and concentrated in vacuo. Column chromatography (5:1 hexanes : EtOAc) gave the desired alkene $23(177 \mathrm{mg}, 44 \%)$ as a yellow oil. Further purification by PTLC (5:1 hexanes : EtOAc) afforded a amorphous solid which was analyzed by HRMS: mp 68-70 ${ }^{\circ} \mathrm{C} ;{ }^{1} \mathrm{H}-\mathrm{NMR}(600 \mathrm{MHz}$, $\left.\mathrm{CDCl}_{3}\right) \delta 8.41(\mathrm{~s}, 1 \mathrm{H}), 7.86(\mathrm{~s}, 1 \mathrm{H}), 7.58-7.60(\mathrm{~m}, 1 \mathrm{H}), 7.33-7.34(\mathrm{~m}, 1 \mathrm{H}), 7.18-7.20(\mathrm{~m}, 1 \mathrm{H}), 7.14-7.15(\mathrm{~d}, 2 \mathrm{H}, \mathrm{J}$ $8.66 \mathrm{~Hz}) 7.10-7.13(\mathrm{~m}, 1 \mathrm{H}), 6.68-6.70(\mathrm{~d}, 2 \mathrm{H}, J 8.66 \mathrm{~Hz}) 6.56-6.57(\mathrm{~m}, 1 \mathrm{H}) 3.84(\mathrm{~s}, 3 \mathrm{H}), 0.96(\mathrm{~s}, 9 \mathrm{H}), 0.19(\mathrm{~s}, 6 \mathrm{H})$; ${ }^{13} \mathrm{C}-\mathrm{NMR}\left(150 \mathrm{MHz}_{\mathrm{CDCl}}\right) \delta 168.1,157.4,142.2,136.2,132.0,131.3,128.5,127.6,122.4,121.4,121.0,120.3$, 120.0, 111.2, 104.7, 52.6, 25.7, 18.3, -4.3; IR $v(\mathrm{KBr}) 3441,1636,1250,1142,902 \mathrm{~cm}^{-1}$; UV $\lambda_{\max }(\mathrm{MeOH}) 350$, 300, $288 \mathrm{~nm}$. HRMS calcd for $\mathrm{C}_{24} \mathrm{H}_{29} \mathrm{NO}_{3} \mathrm{Si}\left(\mathrm{M}^{+}+\mathrm{H}\right)$ 408.1995. Found 408.2008.

(E)-methyl 2-(3-formyl-1H-indol-2-yl)-3-(4-hydroxyphenyl)acrylate (24). $\mathrm{POCl}_{3}(0.109 \mathrm{~g}, 0.07 \mathrm{~mL}, 0.712 \mathrm{mmol}$, 1.2 equiv.) was added to DMF ( $0.052 \mathrm{~g}, 0.05 \mathrm{~mL}, 0.71 \mathrm{mmol}, 1.2$ equiv.) and after stirring for 15 min, alkene 23 $(0.242 \mathrm{~g}, 0.595 \mathrm{mmol}, 1$ equiv.) in 1,2-dichloroethane $(7 \mathrm{~mL})$ was added. The reaction was heated to reflux for $1 \mathrm{~h}$ before it was poured onto an aqueous solution $(1 \mathrm{~mL})$ of $\mathrm{NaOAc}(0.464 \mathrm{~g}, 5.64 \mathrm{mmol}, 9.5$ equiv.) under icecooling and stirred overnight. The reaction mixture was diluted with $\mathrm{H}_{2} \mathrm{O}(15 \mathrm{~mL})$, the aqueous mixture was extracted by $\mathrm{CH}_{2} \mathrm{Cl}_{2}(3 \times 15 \mathrm{~mL})$ and the combined organic extracts were dried $\left(\mathrm{MgSO}_{4}\right)$ and concentrated in vacuo. Flash column chromatography (1:1 hexanes : EtOAc) gave the desired product $24(0.151 \mathrm{~g}, 79 \%)$ as yellow solid; mp $203{ }^{\circ} \mathrm{C}$ (dec); ${ }^{1} \mathrm{H}-\mathrm{NMR}\left(600 \mathrm{MHz}, \mathrm{DMSO}-\mathrm{d}_{6}\right) \delta 12.27(\mathrm{~s}, 1 \mathrm{H}), 10.19(\mathrm{~s}, 1 \mathrm{H}), 9.70(\mathrm{~s}, 1 \mathrm{H}), 8.10-$ $8.12(\mathrm{~m}, 2 \mathrm{H}), 7.48-7.50(\mathrm{~d}, 1 \mathrm{H}, J 7.9 \mathrm{~Hz}), 7.26-7.29(\mathrm{~m}, 2 \mathrm{H}), 6.93-6.95(\mathrm{~d}, 2 \mathrm{H}, J 8.8 \mathrm{~Hz}), 6.60-6.62(\mathrm{~d}, 2 \mathrm{H}, J 8.8$ $\mathrm{Hz}), 3.72(\mathrm{~s}, 3 \mathrm{H}) ;{ }^{13} \mathrm{C}-\mathrm{NMR}\left(150 \mathrm{MHz}, \mathrm{DMSO}_{-} \mathrm{d}_{6}\right)$ 184.5, 166.3, 160.1, 146.2, 144.0, 136.3, 132.6, 125.0, 123.9, 123.6, 122.4., 120.9, 116.4, 115.8, 113.9, 112.3, 52.4; IR $v(\mathrm{KBr}) 3310,2847,1690,1636,1204,748 \mathrm{~cm}^{-1}$; UV $\lambda_{\max }(\mathrm{MeOH}) 213,245,268,303 \mathrm{~nm}$.

(E)-tert-butyl-2-(1-(4-((tert-butoxycarbonyl)oxy)phenyl)-3-methoxy-3-oxoprop-1-en-2-yl)-3-formyl-1H-indole -1-carboxylate (25). To a stirred solution of compound 24 (61 mg $0.168 \mathrm{mmol}, 1$ equiv.) and DMAP (2.3 mg, $0.019 \mathrm{mmol}, 0.1$ equiv.) at $0{ }^{\circ} \mathrm{C}$ in $\mathrm{CH}_{2} \mathrm{Cl}_{2}(5 \mathrm{~mL})$ was added $\mathrm{Boc}_{2} \mathrm{O}$ (102.5 mg, $0.47 \mathrm{mmol}, 2.5$ equiv.). The stirring continued for $4 \mathrm{~h}$ at $\mathrm{rt}$ before the reaction mixture was extracted with $\mathrm{CH}_{2} \mathrm{Cl}_{2}(3 \times 10 \mathrm{~mL})$, quenched with dilute $\mathrm{HCl}$ and washed with $\mathrm{H}_{2} \mathrm{O}$. Evaporation of solvent afforded 25 as a crude white solid (98 mg, 100\%) that was used without further purification: $\mathrm{mp} 102-105{ }^{\circ} \mathrm{C} ;{ }^{1} \mathrm{H}-\mathrm{NMR}\left(600 \mathrm{MHz}, \mathrm{CDCl}_{3}\right) \delta 9.81(\mathrm{~s}, 1 \mathrm{H}), 8.31-8.32$ $(\mathrm{d}, 1 \mathrm{H}, J 7.8 \mathrm{~Hz}), 8.27-8.28(\mathrm{~d}, 1 \mathrm{H}, J 8.3 \mathrm{~Hz}) 8.14(\mathrm{~s}, 1 \mathrm{H}), 7.44-7.46(\mathrm{~m}, 1 \mathrm{H}), 7.38-7.40(\mathrm{~m}, 1 \mathrm{H}), 7.13-7.15(\mathrm{~d}, 2 \mathrm{H}, J$ $8.6 \mathrm{~Hz}), 7.04-7.05(\mathrm{~d}, 2 \mathrm{H}, \mathrm{J} 8.6 \mathrm{~Hz}), 3.78(\mathrm{~s}, 3 \mathrm{H}), 1.57(\mathrm{~s}, 9 \mathrm{H}), 1.50(\mathrm{~s}, 9 \mathrm{H}) ;{ }^{13} \mathrm{C}-\mathrm{NMR}\left(150 \mathrm{MHz}, \mathrm{CDCl}_{3}\right) \delta 186.7$, 166.2, 152.5, 151.2, 149.2, 143.5, 143.4, 136.5, 131.5, 130.7, 126.2, 125.8, 124.9, 122.4, 122.0, 121.9, 118.2, 115.6, 86.4, 84.2, 52.8, 27.9, 27.1; IR $v(\mathrm{KBr})$ 2947, 2847, 1751, 1667, 1543, 1373, $756 \mathrm{~cm}^{-1}$; UV $\lambda_{\max }(\mathrm{MeOH})$ $218,314 \mathrm{~nm}$.

(E)-1-(tert-butoxycarbonyl)-2-(1-(4-((tert-butoxycarbonyl)oxy)phenyl)-3-methoxy-3-oxoprop-1-en-2-yl)-1Hindole-3-carboxylic acid (26). To a heterogeneous mixture of aldehyde 25 (100 mg, $0.19 \mathrm{mmol}, 1$ equiv.) and 
sulfamic acid (75 mg, $0.77 \mathrm{mmol}, 4$ equiv.) in $t-\mathrm{BuOH}: \mathrm{H}_{2} \mathrm{O}(3: 1=4 \mathrm{~mL})$ were added $\mathrm{NaH}_{2} \mathrm{PO}_{4}(69 \mathrm{mg}, 0.58$ mmol, 3 equiv.) and $\mathrm{NaClO}_{2}(80 \%, 73 \mathrm{mg}, 0.81 \mathrm{mmol}, 3$ equiv.), sequentially at room temperature. The reaction mixture was allowed to stir for $8 \mathrm{~h}$ then poured onto $\mathrm{H}_{2} \mathrm{O}(10 \mathrm{~mL})$ and extracted with EtOAc $(3 \times 10$ $\mathrm{mL})$. The combined organic extracts were concentrated in vacuo and purified by flash column chromatography (1:1 hexanes : EtOAc) to furnish acid 26 as a white solid (89 mg, 87\%): mp 144-146 ${ }^{\circ} \mathrm{C} ;{ }^{1} \mathrm{H}-\mathrm{NMR}(600 \mathrm{MHz}$, $\left.\mathrm{CDCl}_{3}\right) \delta 8.23-8.27(\mathrm{~m}, 2 \mathrm{H}), 7.95(\mathrm{~s}, 1 \mathrm{H}), 7.38-7.44(\mathrm{~m}, 2 \mathrm{H}), 6.97-7.01(\mathrm{~m}, 4 \mathrm{H}), 3.76(\mathrm{~s}, 3 \mathrm{H}), 1.49(\mathrm{~s}, 9 \mathrm{H}), 1.48(\mathrm{~s}$, $9 \mathrm{H}) ;{ }^{13} \mathrm{C}-\mathrm{NMR}\left(150 \mathrm{MHz}, \mathrm{CDCl}_{3}\right) \delta 168.7,166.6,152.1,151.3,149.0,141.6,139.9,136.2,131.6,130.9,127.3$, 125.6, 124.4, 124.1, 122.4, 121.7, 115.6, 111.3, 85.9, 84.0, 52.6, 27.9, 27.7; IR v (KBr) 2978, 2932, 1751, 1674, $1373,1150 \mathrm{~cm}^{-1}$; UV $\lambda_{\max }(\mathrm{MeOH}) 271,272,282 \mathrm{~nm}$.

(E)-2-(1-(4-hydroxyphenyl)-3-methoxy-3-oxoprop-1-en-2-yl)-1H-indole-3-carboxylic acid (3). To a solution of di-Boc acid 26 (49.7 mg, $0.925 \mathrm{mmol}, 1$ equiv.) in $\mathrm{CH}_{2} \mathrm{Cl}_{2}(2 \mathrm{~mL})$ at $0{ }^{\circ} \mathrm{C}$ was added TFA (0.55 mL). The mixture was allowed to stir for $8 \mathrm{~h}$ before being concentrated by rotary evaporation to give the crude product. Purification by PTLC (1:2 hexanes : EtOAc) afforded the pure compound 3 as a yellow oil $(7.6 \mathrm{mg}, 24 \%):{ }^{1} \mathrm{H}$ NMR $(600 \mathrm{MHz}, \text { DMSO-d })_{6} \delta 11.84(\mathrm{~s}, 1 \mathrm{H}), 10.05(\mathrm{~s}, 1 \mathrm{H}), 8.04-8.05(\mathrm{~d}, 1 \mathrm{H}, J 6.7 \mathrm{~Hz}), 7.79(\mathrm{~s}, 1 \mathrm{H}), 7.37-7.38(\mathrm{~d}$, $1 \mathrm{H}$, J $6.7 \mathrm{~Hz}), 7.18-7.20(\mathrm{~m}, 2 \mathrm{H}), 6.84-6.85(\mathrm{~d}, 2 \mathrm{H}, J 8.3 \mathrm{~Hz}), 6.58-6.59(\mathrm{~d}, 2 \mathrm{H}, J 8.3 \mathrm{~Hz}), 3.64(\mathrm{~s}, 3 \mathrm{H}) ;{ }^{13} \mathrm{C}-\mathrm{NMR}$ $\left(150 \mathrm{MHz}, \mathrm{DMSO}-\mathrm{d}_{6}\right) \delta 166.8,165.7,159.4$ 142.1, 139.2, 135.7, 132.2, 127.0, 124.7, 122.4, 121.1, 121.1, 120.3, 115.6, 112.0, 105.7, 52.0; IR $v(\mathrm{KBr}) 3549,2924,2855,1643,1574,1512,1443 \mathrm{~cm}^{-1}$; UV $\lambda_{\max }(\mathrm{MeOH}) 231,286$, $321 \mathrm{~nm}$.

\section{Acknowledgements}

This work was supported by the School of Graduate Studies and Research, University of the West Indies, Cave Hill, and the Government of Barbados. The authors thank Prof. Gordon Gribble for his continued support, encouragement and mentorship.

\section{References}

1. Klein-Júnior, L. ; Cretton, S.; Allard, P-M.; Genta-Jouve, G.; Passos, C.; Salton, J.; Bertelli, P.; Pupier, M.;Jeannerat, D.; Heyden, Y. V.; Gasper, A.; Wolfender, J-L.; Christen, P.; Henriques, A. J. Nat. Prod. 2017, 80, 3032-3037.

https://doi.org/10.1021/acs.jnatprod.7b00681

2. Liu, L.; Wang, L.; Bao, L.; Ren, J.; Bahadur Basnet, B.; Liu, R.; He, L.; Han, J.; Yin, W-B.; Liu, H. Org. Lett. 2017, 19, 942-945.

https://doi.org/10.1021/acs.orglett.7b00145

3. Burja, A. M.; Banaigs, B.; Abou-Mansour, E.; Grant Burgess, J.; Wright, P. C. Tetrahedron 2001, 57, 93479377. https://doi.org/10.1016/S0040-4020(01)00931-0

4. Singh, R. K.; Tiwari, S. P.; Rai, A. K.; Mohapatra, T. J. Antibiot. 2011, 64, 401-412. https://doi.org/10.1038/ia.2011.21

5. Skulberg, O. J. Appl. Phycol. 2000, 12, 341-348.

https://doi.org/10.1023/A:1008140403621 
6. Schopf, J. W.; Packer, B. M. Science 1987, 237, 70-31.

https://doi.org/10.1126/science.11539686

7. Garcia-Pichel, F.; López-Cortés, A.; Nübel, U. Appl. Environ. Microbiol. 2001, 1902-1910. https://doi.org/10.1128/AEM.67.4.1902-1910.2001

8. Taton, A.; Grubisic, S.; Brambilla, E.; De Wit, R.; Wilmotte, A. Appl. Environ. Microbiol. 2003, 5157-5169. https://doi.org/10.1128/AEM.69.9.5157-5169.2003

9. Vaillancourt, V.; Albizati, K. F. J. Am. Chem. Soc. 1993, 115, 3499-3502. https://doi.org/10.1021/ja00062a013

10. Baran, P. S.; Maimone, T. J.; Richter, J. M. Nature 2007, 446, 404-408. https://doi.org/10.1038/nature05569

11. Muratake, H.; Natsume, M. Tetrahedron 1990, 46, 6331-6342. https://doi.org/10.1016/S0040-4020(01)96005-3

12. Muratake, H.; Natsume, M. Tetrahedron 1990, 46, 6343-6350. https://doi.org/10.1016/S0040-4020(01)96006-5

13. Muratake, H.; Kumagami, H.; Natsume, M. Tetrahedron 1990, 46, 6351-6360. https://doi.org/10.1016/S0040-4020(01)96007-7

14. Rafferty, R. J.; Williams, R. M. J. Org. Chem. 2011, 77, 519-524. https://doi.org/10.1021/jo202139k

15. Fukuyama, T.; Chen, X. J. Am. Chem. Soc. 1994, 116, 3125-3126. https://doi.org/10.1021/ja00086a053

16. Chandra, A.; Johnston, J. N. Angew. Chem. Int. Ed. 2011, 50, 7641-7644. https://doi.org/10.1002/anie.201100957

17. Baran, P. S.; Richter, J. M. J. Am. Chem. Soc. 2004, 126, 7450-7451. https://doi.org/10.1021/ja047874w

18. Richter, J. M.; Ishihara, Y.; Masuda, T.; Whitefield, B. W.; Llamas, T. S.; Pohjakallio, A.; Baran, P. S. J. Am. Chem. Soc. 2008, 130, 17938-17954. https://doi.org/10.1021/ja806981k

19. Kinsman, A. C.; Kerr, M. A. Org. Lett. 2001, 3, 3189-3191. https://doi.org/10.1021/ol0165138

20. Sakagami, M.; Muratake, H.; Natsume, M. Chem. Pharm. Bull. 1994, 42, 1393-1398. https://doi.org/10.1248/cpb.42.1393

21. Muratake, H.; Natsume, M. Tetrahedron Lett. 1989, 30, 1815-1818. https://doi.org/10.1016/S0040-4039(00)99587-X

22. Baran, P. S.; Richter, J. M. J. Am. Chem. Soc. 2005, 127, 15394-15396. https://doi.org/10.1021/ja056171r

23. Chandra, A.; Viswanathan, R.; Johnston, J. N. Org. Lett. 2007, 9, 5027-5029. https://doi.org/10.1021/ol702247a

24. Rafferty, R. J.; Williams, R. M. Tetrahedron Lett. 2011, 52, 2037-2040. https://doi.org/10.1016/j.tetlet.2010.09.086

25. Reisman, S. E.; Ready, J. M.; Hasuoka, A.; Smith, C. J.; Wood, J. L. J. Am. Chem. Soc. 2006, 128, 1448-1449. https://doi.org/10.1021/ja057640s

26. Allan, K. M.; Kobayashi, K.; Rawal, V. H. J. Am. Chem. Soc. 2011, 134, 1392-1395. https://doi.org/10.1021/ja210793x 
27. Bhat, V.; Rawal, V. H. Chem. Commun. 2011, 47, 9705-9707. https://doi.org/10.1039/c1cc13498a

28. Bhat, V.; Allan, K. M.; Rawal, V. H. J. Am. Chem. Soc. 2011, 133, 5798-5801. https://doi.org/10.1021/ja201834u

29. Quasdorf, K. W.; Huters, A. D.; Lodewyk, M. W.; Tantillo, D. J.; Garg, N. K. J. Am. Chem. Soc. 2011, 134, 1396-1399.

https://doi.org/10.1021/ja210837b

30. Moore, R. E.; Cheuk, C.; Patterson, G. M. L. J. Am. Chem. Soc. 1984, 106, 6456-6457. https://doi.org/10.1021/ja00333a079

31. Proteau, P. J.; Gerwick, W. H.; Garcia-Pichel, F.; Castenholz, R. Experientia 1993, 49, 825-829. https://doi.org/10.1007/BF01923559

32. Kobayashi, A.; Kajiyama, S.-i.; Inawaka, K.; Kanzaki, H.; Kawazu, K. J. Biosci. 1994, 49c, 464-470.

33. Ploutno, A.; Carmeli, S. J. Nat. Prod. 2001, 64, 544-545. https://doi.org/10.1007/BF01923559

34. Shim, S. H.; Chlipala, G.; Orjala, J. J. Microbiol. Biotechnol. 2008, 18, 1655-1658.

35. Ekebergh, A.; Karlsson, I.; Mete, R.; Pan, Y.; Börje, A.; Mårtensson, J. Org. Lett. 2011, 13, 4458-4461. https://doi.org/10.1021/ol201812n

36. Ekebergh, A.; Börje, A.; Mårtensson, J. Org. Lett. 2012, 14, 6274-6277. https://doi.org/10.1021/ol303036j

37. Biswas, S.; Jaiswal, P. K.; Singh, S.; Mobin, S. M.; Samanta, S. Org. Biomol. Chem. 2013, 11, 7084-7087. https://doi.org/10.1039/c3ob41573b

38. McNulty, J.; Keskar, K.; Bordon, C.; Yolken, R.; Jones-Brando, L. Chem. Commun. 2014, 50, 8904-8907. https://doi.org/10.1039/C4CC03904A

39. McNulty, J.; Keskar, K.; Jenkins, H.; Werstiuk, N.; Bordon, C.; Yolken, R.; Jones-Brando, L. Org. Biomol. Chem. 2015, 13, 10015-10024. https://doi.org/10.1039/C5OB01506E

40. Balskus, E. P.; Walsh, C. T. J. Am. Chem. Soc. 2008, 130, 15260-15261. https://doi.org/10.1021/ja807192u

41. Zhang, G.; Zhang, Z.; Liu, Z. Tumor Biol. 2013, 1-7.

42. Chui-ze, Z. Z. K. J. China Med. Univ. 2011, 40, 844-845, 847.

43. Soule, T.; Stout, V.; Swingley, W. D.; Meeks, J. C.; Garcia-Pichel, F. J. Bacteriol. 2007, 189, 4465-4472. https://doi.org/10.1128/JB.01816-06

44. Stevenson, C. S.; Capper, E. A.; Roshak, A. K.; Marquez, B.; Eichman, C.; Jackson, J. R.; Mattern, M.; Gerwick, W. H.; Jacobs, R. S.; Marshall, L. A. J. Pharmacol. Exp. Ther. 2002, 303, 858-866. https://doi.org/10.1124/ipet.102.036350

45. Stevenson, C. S.; Capper, E. A.; Roshak, A. K.; Marquez, B.; Grace, K.; Gerwick, W. H.; Jacobs, R. S.; Marshall, L. A. Inflamm. Res. 2002, 51, 112-114.

https://doi.org/10.1007/BF02684014

46. Garcia-Pichel, F.; Castenholz, R. W. J. Phycol. 1991, 27, 395-409.

https://doi.org/10.1111/i.0022-3646.1991.00395.x

47. Balskus, E. P.; Walsh, C. T. J. Am. Chem. Soc. 2009, 131, 14648-14649. https://doi.org/10.1021/ia906752u

48. Balskus, E. P.; Case, R. J.; Walsh, C. T. FEMS Microbiol. Ecol. 2011, 77, 322-332. https://doi.org/10.1111/j.1574-6941.2011.01113.x 
49. Bultel-Poncé, V.; Felix-Theodose, F.; Sarthou, C.; Ponge, J. F.; Bodo, B. J. Nat. Prod. 2004, 67, 678-681. https://doi.org/10.1021/np034031u

50. Grant, C. S.; Louda, J. W. Org. Geochem. 2013, 65, 29-36. https://doi.org/10.1016/j.orggeochem.2013.09.014

51. Badenock, J. C.; Jordan, J. A.; Gribble, G. W. Tetrahedron Lett. 2013, 54, 2759-2762. https://doi.org/10.1016/j.tetlet.2013.02.116

52. Compound $\mathbf{1 2}$ resulting from coupling of methyl ester $\mathbf{1 1}$ with $\mathbf{1 4}$ was observed to possess an E-geometry around the exocyclic double bond while a similar coupling of the ethyl ester analogue of 11 with 14 generated a vinyl indole with the Z-geometry.

53. Bahadur, G. A.; Bailey, A. S.; Middleton, N. W.; Peach, J. M. J. Chem. Soc., Perkin Trans. 1 1980, 1688-1692. https://doi.org/10.1039/p19800001688

54. Macor, J. E.; Newman, M. E.; Ryan, K. Tetrahedron Lett. 1989, 30, 2509-2512. https://doi.org/10.1016/S0040-4039(01)80437-8

55. James, P. N.; Snyder, H. R. Org. Synth. 1959, 39, 30.

https://doi.org/10.15227/orgsyn.039.0030

56. Coulton, S.; L. Gilchrist, T.; Graham, K. J. Chem. Soc., Perkin Trans. 1 1998, 1193-1202. https://doi.org/10.1039/a800278i

57. Cleavage of the triphenyl phosphine group alone resulted when the recommended three equivalents of base were used in the Wittig reaction.

58. Green, I. L. MPhil. Thesis, Univ. of the West Indies, Cave Hill, Barbados, 2015.

59. Yoshikawa, K.; Yokomizo, A.; Naito, H.; Haginoya, N.; Kobayashi, S.; Yoshino, T.; Nagata, T.; Mochizuki, A.; Osanai, K.; Watanabe, K.; Kanno, H.; Ohta, T. Bioorg. Med. Chem. 2009, 17, 8206-8220. https://doi.org/10.1016/j.bmc.2009.10.023

60. Lindgren, B. O.; Nilsson, T. Acta Chem. Scand. 1973, 27, 888-890. https://doi.org/10.3891/acta.chem.scand.27-0888

61. S. Bal, B.; Childers Jr, W.; Pinnick, H. Tetrahedron 1981, 37, 2091-2096. https://doi.org/10.1016/S0040-4020(01)97963-3

62. Mahboobi, S.; Bernauer, K. Helv. Chim. Acta 1988, 71, 2034-2041. https://doi.org/10.1002/hlca.19880710821 